\title{
Uso de Realidade Aumentada como Apoio ao Ensino do Campo Girante de um Motor de Corrente Alternada
}

\author{
Using Augmented Reality to support teaching the rotating field of an alternating cur- \\ rent motor
}

\author{
Suzana da Hora Macedo \\ Instituto Federal Fluminense \\ shmacedo@iff.edu.br \\ Evanildo dos Santos Leite \\ Instituto Federal Fluminense \\ eleite@iff.edu.br
}

\author{
Filipe Arantes Fernandes \\ Instituto Federal Fluminense \\ filran@gmail.com \\ José Valdeni de Lima \\ Universidade Federal do Rio Grande do Sul \\ valdeni@inf.ufrgs.br
}

Maria Cristina Villanova Biazus

Universidade Federal do Rio Grande do Sul

cbiazus@ufrgs.br

\begin{abstract}
Resumo Este estudo tem como objetivo melhorar o processo de ensino-aprendizagem dos conceitos de Eletromagnetismo usando Realidade Aumentada (RA). Eletromagnetismo é a base científica para a compreensão do princípio de funcionamento das máquinas elétricas rotativas. No entanto, verificouse através de um estudo preliminar que existem dificuldades no processo de ensino-aprendizagem de Eletromagnetismo e que estas dificuldades estão concentradas basicamente em dois fatores: na visualização da representação correta do campo magnético e na base matemática para trabalhar com esses conceitos, os quais permitiriam a aquisição de novos conceitos na área de Eletromagnetismo. Neste trabalho, foi desenvolvido e utilizado um Objeto de Aprendizagem como ferramenta no processo de ensino-aprendizagem de conceitos de Eletromagnetismo para se superar o obstáculo da visualização dos campos magnéticos. Este Objeto de Aprendizagem foi testado com os alunos dos cursos técnicos do Instituto Federal Fluminense (IFF), onde o aluno pode visualizar e interagir com os mesmos. Este trabalho foi baseado na teoria da Aprendizagem Significativa, que, de acordo com Ausubel, ocorre quando um conceito está relacionado de forma significativa e não arbitrária, com conceitos preexistentes na estrutura cognitiva do indivíduo. A partir dos resultados obtidos com a interação dos alunos com os Objetos de Aprendizagem foi possivel fazer análises e avaliações que permitiram concluir o presente trabalho. A análise dos resultados mostra que houve melhorias significativas no desempenho dos aprendizes do grupo experimental, quando comparados aos aprendizes do grupo de controle. Comentários e avaliações foram feitos para complementar o trabalho, destacando suas vantagens no processo de ensino-aprendizagem.
\end{abstract}

Palavras-Chave: Realidade Aumentada, campo girante, motor, Aprendizagem Significativa.

\footnotetext{
Abstract

This work aims to improve teaching and learning Electromagnetism concepts using Augmented Reality (AR). Electromagnetism is the scientific basis for understanding the principle of rotating electrical machines. However, it was verified through a preliminary study that there are difficulties in the Electromagnetism teaching- learning process and that these difficulties are concentrated primari-
} 
ly on two factors: visualize the representation of the magnetic field and the mathematical basis to work with these concepts, which allow the acquisition of new concepts in the field of Electromagnetism. In this work, it was developed and used a learning object as a tool in the teaching-learning of Electromagnetism concepts to overcome the dificulties of the process visualization of magnetic fields. This Learning Object was tested with students of technical courses at Instituto Federal Fluminense (IFF), where students can view and interact with them. This work was based on the theory of Meaningful Learning, which, according to Ausubel, occurs when a concept is related significantly and not arbitrary, with existing concepts in the cognitive structure of the individual. From the results obtained with the interaction of students with the Learning Objects was possible to make analyzes and reviews to complete the present work. The analysis shows that there were significant improvements in the performance of learners in the experimental group compared to the control group learners. Comments and reviews were made to complement the work, highlighting its advantages in the teaching- learning process.

Keywords: Augmented Reality, rotating magnetic field, motor, Meaningful Learning. 


\section{Introdução}

A conversão direta da energia elétrica em mecânica só é possível graças à interação de campos magnéticos rotativos cuja base científica é o Eletromagnetismo, sendo estes, conceitos de fundamental importância na formação dos alunos que estudam Eletricidade.

Parte das dificuldades de aprendizagem dos conteúdos de Eletromagnetismo se concentra na impossibilidade de visualização dos campos magnéticos, por parte dos alunos, no espaço tridimensional. Segundo Paz [1], "constatamos que as dificuldades de aprendizagem dos conteúdos de Eletromagnetismo se concentram no entendimento das interações e comportamento das variáveis eletromagnéticas no espaço tridimensional".

Macedo et al. [2], afirmam que com o advento do computador novos espaços de aprendizagem começam a ser planejados e construídos e estes espaços não estão mais restritos ao perímetro em que ocorre uma relação tradicional e fechada entre professores e alunos. Segundo Buchau et al [3], a Realidade Aumentada (RA) ajuda os estudantes a compreenderem a teoria dos campos magnéticos, sendo muito adequada para visualização destes campos.

Silva et al. [4] Afirmam que "os sistemas virtuais possibilitam experiências com a sensação de presença, por meio da integração dinâmica, de diferentes modalidades perceptivas, que envolvem imagens, sons, tato, etc".

Objeto de Aprendizagem (OA) é qualquer recurso digital que pode ser reutilizado para apoiar a aprendizagem [5]. Neste contexto, foi proposta a criação de um OA desenvolvido com recursos de RA.

Segundo Barros [6], "A cada tema o usuário vai participar do ambiente virtual gerado pelo computador,.... neste caso, o ambiente virtual será projetado para simular tanto um ambiente imaginário quanto um ambiente real".

Macedo e Leite [7] em seu trabalho que utiliza um simulador para o ensino de Eletrônica Digital, afirmam sobre os computadores, que "a sua utilização na escola como recurso no processo de ensino-aprendizagem é de grande valia". Com este OA será simulado um ambiente que é impossível de ser visto a olho nu: o campo magnético. Campos eletromagnéticos invisíveis no ar podem ser mostrados em ambiente de RA [3]. A RA mistura o mundo real com o mundo virtual. Na tela do computador, o aluno poderá ver o mundo real misturado com o mundo virtual, possibilitando assim que o mesmo visualize o ímã e seu campo magnético em uma mesma tela do computador. O mundo real será composto de ímãs e o mundo virtual será composto da representação pictórica dos campos magnéticos gerados por estes ímãs e suas interações. Segundo Coelho e Bähr [8], "A combinação de real com virtual se baseia na presença simultânea de objetos reais e virtuais em uma cena". No caso deste experimento, o objeto real será o motor, e o mundo virtual será o campo girante que surgirá ao redor do mesmo. Os objetos reais e virtuais aparecerão na tela do computador. A RA servirá para ampliar o horizonte do aluno, possibilitando a interação com o fenômeno físico em estudo, fazendo com que ele visualize no mundo virtual objetos que os seres humanos não conseguem visualizar no mundo real.

Segundo Silva et al. [4], a possibilidade de interação entre objetos reais e virtuais, que ocorre por meio da RA, pode oferecer ao usuário maiores informações sensitivas, facilitando a associação e a reflexão sobre a situação.

Segundo Kaufmann et al. [9], a principal vantagem do uso da Realidade Aumentada é que os alunos realmente veem objetos tridimensionais. Desse modo, os campos magnéticos serão visualizados no mundo virtual, interagindo com os ímãs do mundo real. Este experimento teve como objetivo criar um ambiente onde o aluno pudesse visualizar em terceira dimensão o campo girante de um motor de corrente alternada, além de criar um ambiente lúdico para o estudante.

\section{Algumas Propostas do Uso da Rea- lidade Aumentada na Educação}

Apesar de recente, a Realidade Aumentada já vem sendo utilizada com sucesso em diversas áreas. Segundo Valente [10], "a cada dia surgem novas maneiras de usar o computador como um recurso para enriquecer e favorecer o processo de aprendizagem".

Segundo Coelho e Bähr [8], quando filmamos um local em tempo real e inserimos objetos virtuais e as cenas formadas dão a impressão de que os objetos virtuais existem no mundo real, temos um ambiente de Realidade Aumentada (RA). Diversos pesquisadores estão criando ambientes em Realidade Aumentada com o objetivo de facilitar a aprendizagem. Algumas propostas de uso da Realidade Aumentada na Educação serão abordadas a seguir.

\subsection{MiRa}

O MiRa (Microscópio Simulado em Realidade Aumentada) foi idealizado para facilitar o ensino de Biologia Celular e Tecidual na Educação a Distância por Meio de Microscópio Virtual. 


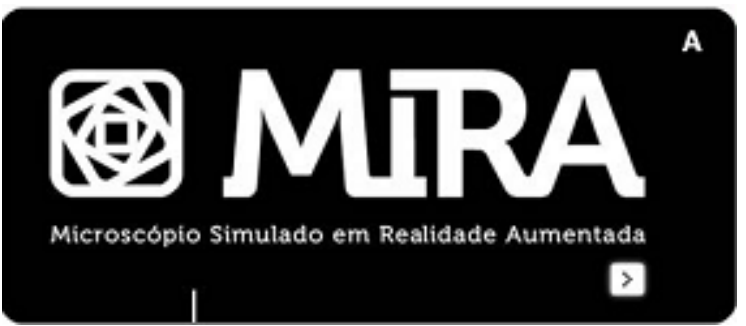

Figura 1 : A Tela de Apresentação do MiRa [11].

A figura 1 mostra a sua tela de apresentação, e a figura 2 mostra a tela do microscópio virtual.

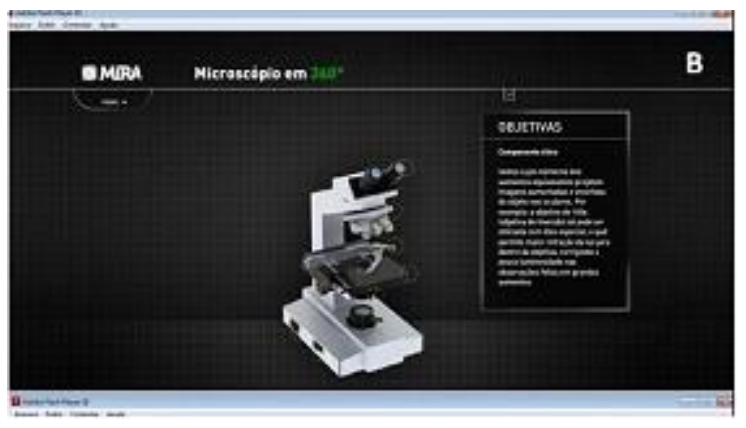

Figura 2 : Microscópio virtual - identificação das objetivas e suas funções [11].

É um software que permite aos estudantes da modalidade EaD (Educação a Distância) do curso de Especialização em Tecnologias Aplicadas ao Ensino de Biologia (ETAEB) pós-graduação lato sensu, na disciplina Fundamentos de Biologia Celular e Tecidual para o Ensino da Universidade Federal de Goiás (UFG), uma leitura, de forma virtual, de lâminas microscópicas por meio de um banco de dados contido nele, que antes só seria possível em um laboratório.

\subsection{Experimento de Oersted}

Souza e Kirner [12] apresentaram uma aplicação de Realidade Aumentada onde o aluno pode fazer experimentos com um circuito elétrico tal como se estivesse em um mini laboratório e ainda com a opção de ser instruído por um tutor em áudio que explica os efeitos da interação sobre o circuito. A figura 3 mostra esse trabalho onde o aluno pode realizar o experimento de Oersted em ambiente de Realidade Aumentada.

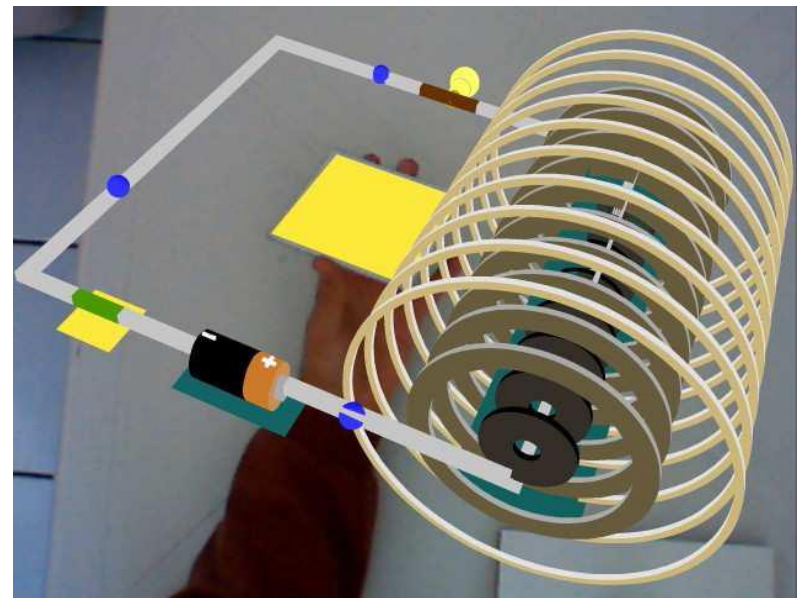

Figura 3 : Experimento de Oersted em Realidade Aumentada Fonte: Souza e Kirner [12]

\subsection{Sistemas Estruturais}

Rodrigues et al. [13] apresentam uma ferramenta de ensino com o intuito de introduzir os alunos no estudo de sistemas estruturais de uma maneira lúdica e adequada ao perfil dos estudantes de Arquitetura da Universidade Federal do Rio de Janeiro.

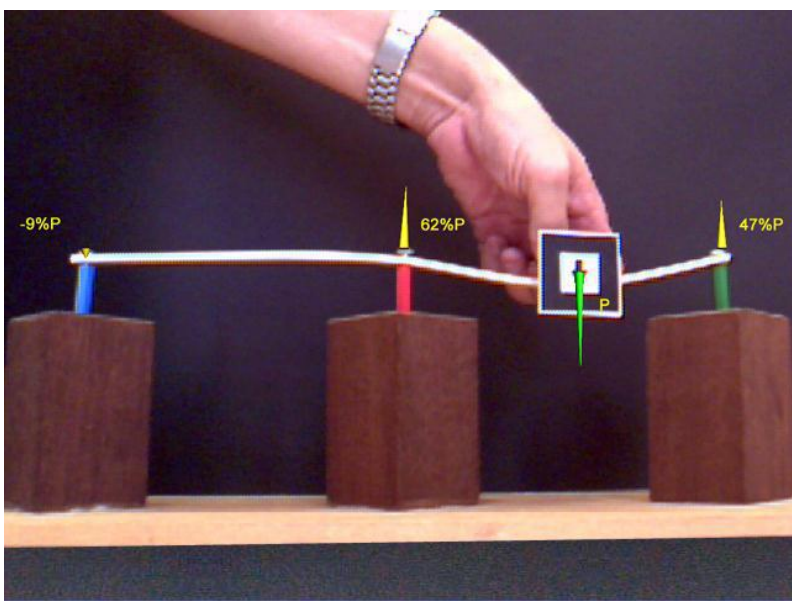

Figura 4 : Reações de apoio da viga contínua [13]

$\mathrm{Na}$ figura 4 pode-se observar as reações de apoio da viga contínua em RA. Este experimento possibilitou aos alunos uma melhor percepção da configuração deformada de sistemas estruturais submetidos a uma carga concentrada em diferentes pontos.

\subsection{Campo Magnético de um Ímã em Forma de Barra}

Macedo et al. [2] estudaram os campos magnéticos de um ímã em forma de barra (figura 5). 


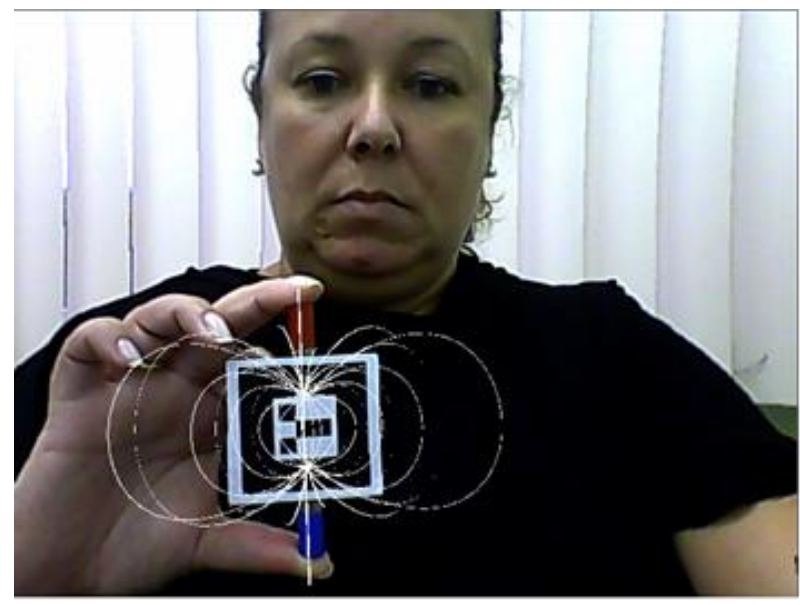

Figura 5 : Campo magnético de um ímã em forma de barra [2]

Em ambiente de Realidade Aumentada os estudantes puderam visualizar em 3D e interagir com o campo magnético de um ímã em forma de barra. A partir dos experimentos realizados com alunos foram feitas análises e avaliações do trabalho.

\subsection{SISEULER}

Também utilizando a Realidade Aumentada no ensino, Lemos e Carvalho [14] criaram o SISEULER, que atua como Objeto de Aprendizagem, onde o aluno pode ter um melhor entendimento da relação de Euler através da visualização e manipulação de objetos. O software SISEULER foi implementado com técnicas de Realidade Aumentada e utilizado para apoio ao ensino da Relação de Euler. O software baseado em Realidade Aumentada permite ao aluno a visualização em terceira dimensão e também a interação com os objetos.

Neste trabalho, a Relação de Euler é utilizada para associar o número de vértices, faces e arestas dos poliedros. Este experimento foi testado com um resultado positivo com professores da educação básica que estão cursando mestrado profissional em Educação Matemática. A figura 6 mostra um dodecaedro utilizando o SIS-EULER.

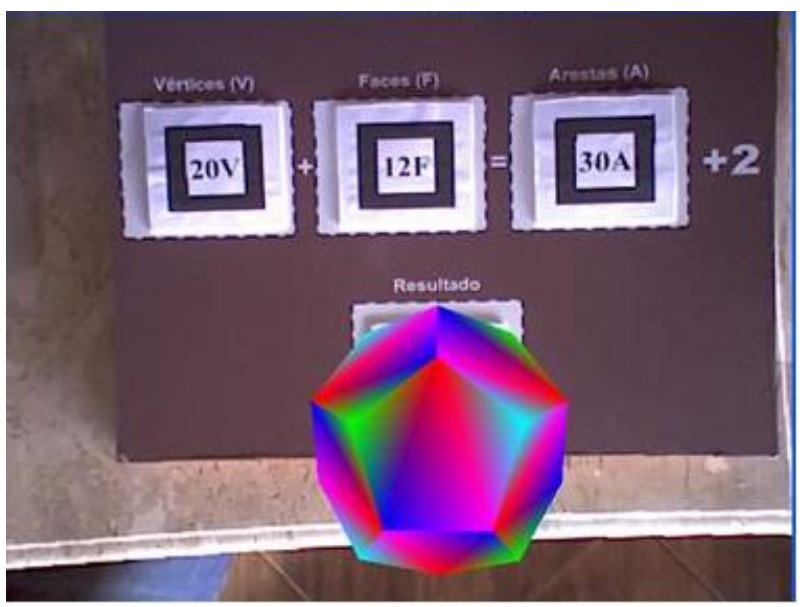

Figura 6 : Dodecaedro no SISEULER criado por Lemos e Carvalho [14]

\subsection{Visualização de Sólidos}

Macedo et al. [15] apresentou um Método de Ensino de Sólidos utilizando Realidade Aumentada, proporcionando ao estudante a interação e visualização dos sólidos.

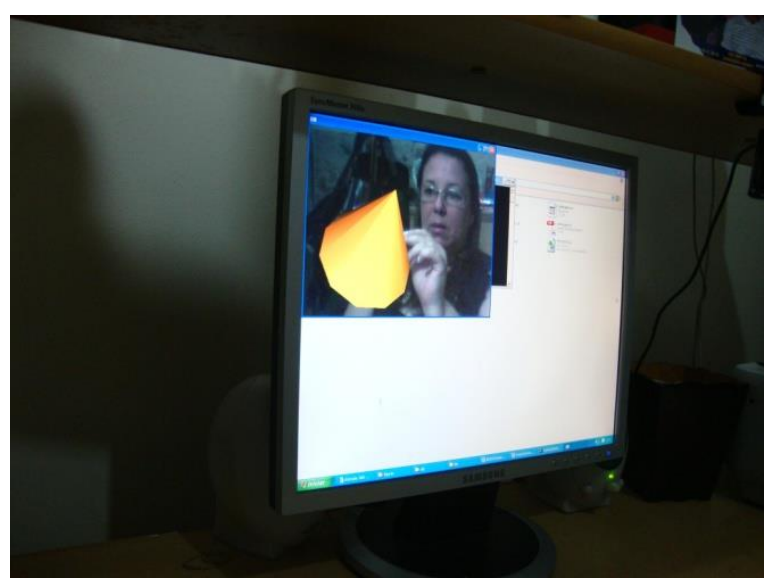

Figura 7 : Usuária manipulando uma pirâmide em Realidade Aumentada [15]

A figura 7 mostra a usuária em um ambiente de Realidade Aumentada manipulando uma pirâmide.

Neste trabalho, diversos sólidos podem ser vistos em terceira dimensão e também podem ser manipulados pelo estudante. A grande vantagem, além da visualização em terceira dimensão e da manipulação é que o custo é baixíssimo, já que, após feita a programação, o equipamento necessário se resume apenas a um laptop ou microcomputador com webcam. 


\subsection{VGSTARGD}

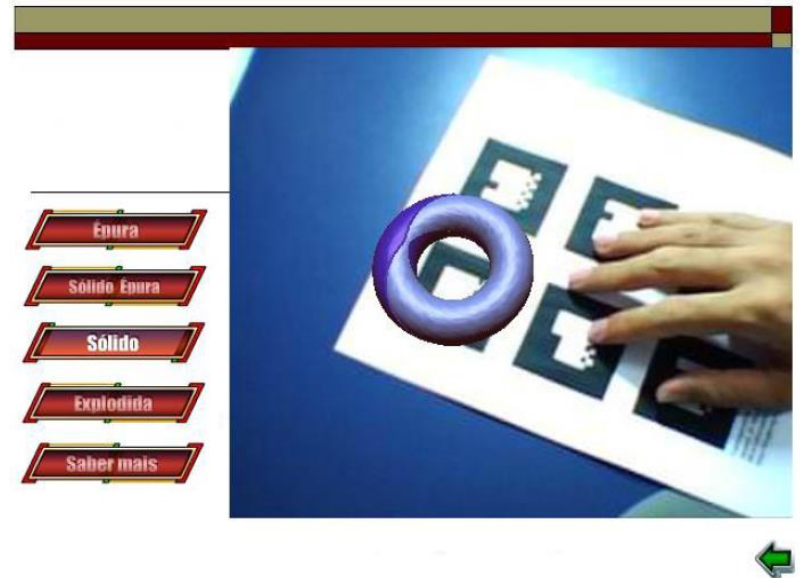

Figura 8 : VGSTARGD desenvolvido pela UFRJ [16]

Lima et al. [16] elaboraram o VSTARGD (Viewer of Torus Surfaces of Descriptive Geometry Through Reality). Neste software se pode visualizar superfícies tóricas, onde três são animadas. A figura 8 mostra a o VSTARGD.

\subsection{Visualização do Campo Magnético de um Solenóide}

Buchau et al. [3] criaram três aplicações baseadas em Realidade Aumentada para serem utilizadas no ensino do campo magnético de um ímã, do campo magnético de um solenóide e do campo magnético de uma antena. $\mathrm{Na}$ figura 9, pode-se ver o campo magnético de um solenóide, resultado deste trabalho.

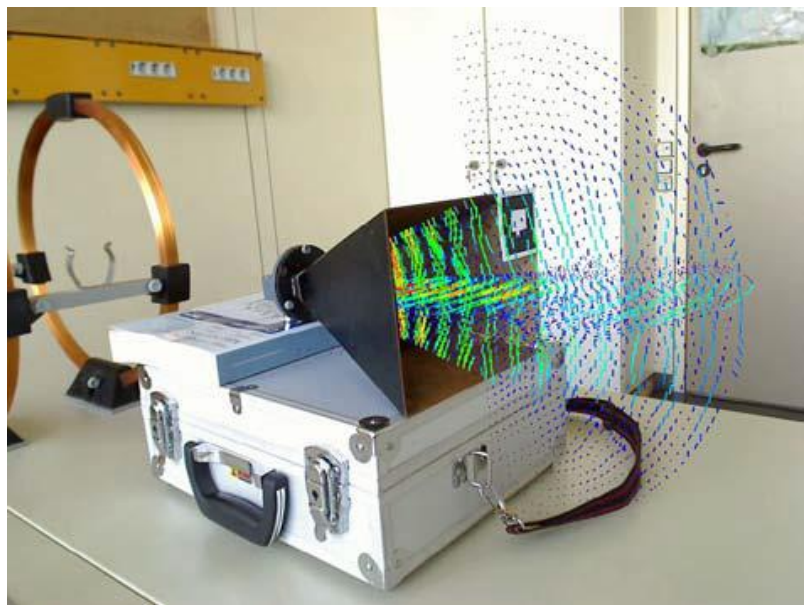

Figura 9 : Campo magnético de uma antena [3]

Estas aplicações permitem que o aluno visualize os campos magnéticos em três dimensões. Este trabalho ainda não apresenta resultados de utilização com estudantes.

\subsection{Construct 3D}

Kaufmann, Steinbüegl, Dünser e Glück [9] da Universidade Tecnológica de Viena desenvolveram o Construct 3D.

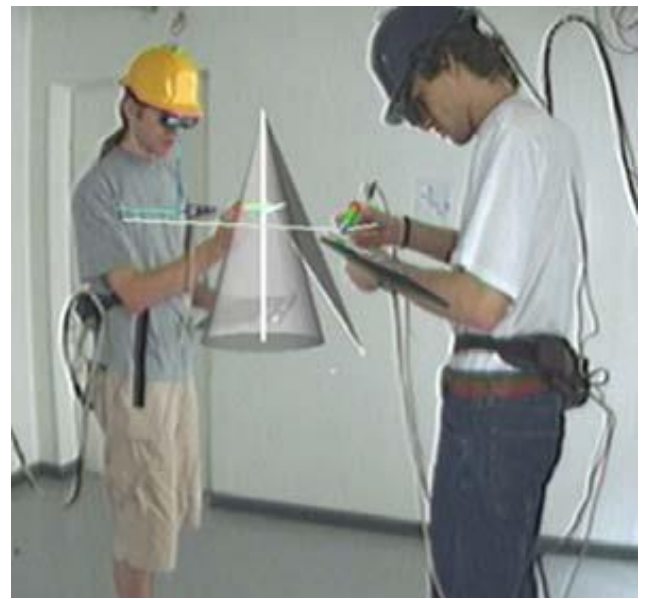

Figura 10 : Estudantes usando o Construct 3D [9]

É um sistema que usa Realidade Aumentada como um meio para o ensino, e usa 3D para facilitar o ensino da matemática e geometria. A figura 10 mostra os estudantes utilizando o Construct 3D. Essa colaboração educacional serve como base de um estudo de avaliação global quanto à eficácia na formação de habilidades espaciais.

\section{O ensino Tradicional do Campo Gi- rante}

O ensino tradicional do campo girante se dá, em geral, através da análise da figura 11 ou uma figura similar.
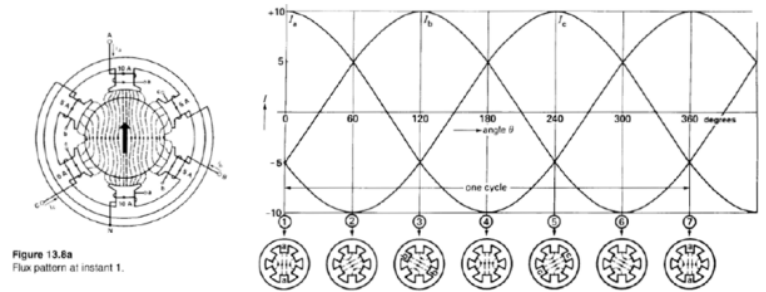

Figura 11 : Imagem utilizada para analisar o campo girante [17]

A figura 11 é normalmente utilizada para se explicar a formação do campo girante trifásico. À esquerda pode-se visualizar os três pares de pólos de um motor e à direita o gráfico mostra as correntes das três fases defasadas de 
$120^{\circ}$. O estudante tem então que tentar imaginar os campos magnéticos criados por cada fase em questão, a resultante do campo magnético gerado e ainda imaginar este campo magnético girando. Note-se que é uma figura bidimensional e estática, explicando um campo girante que na realidade é tridimensional e dinâmico.

Este tipo de estudo conduz o educando a muitas dúvidas, já que é uma visualização rudimentar, em que dificilmente o aluno consegue visualizar este campo magnético girante.

\section{Proposta Pedagógica Baseada na Aprendizagem Significativa}

Esta proposta pedagógica está baseada na Teoria da Aprendizagem Significativa de David Ausubel. "Aprendizagem significativa é um processo pelo qual uma nova informação se relaciona com um aspecto relevante da estrutura de conhecimento do indivíduo" [18]. Neste trabalho, a partir de conceitos já existentes na estrutura cognitiva do aluno, pretende-se que o aluno os utilize como subsunçores de forma a alicerçar os novos conceitos a serem apreendidos. Segundo Moreira [19] "o subsunçor é um conceito, uma idéia, uma proposição já existente na estrutura cognitiva, capaz de servir de 'ancoradouro' a uma nova informação de modo que esta adquira, assim, significado para o indivíduo (isto é, que ele tenha condições de atribuir significados a essa informação)". Nesse aspecto os conceitos previamente "ancorados" na estrutura cognitiva do aluno, servirão como subsunçores, para que uma nova informação seja adquirida por parte do educando. Como nova informação, entende-se os conceitos de Eletromagnetismo necessários ao ensino do campo girante. Guimarães [20] afirma que, para que a Aprendizagem Significativa possa ser observada deverá ser criada "uma situação nova que exija transformação do conhecimento original". A Aprendizagem Significativa foi verificada da seguinte forma: (a) assistiram a uma explicação tradicional sobre a formação do campo girante; (b) foi realizado o experimento em Realidade Aumentada; (c) responderam perguntas sobre o novo conhecimento.

Foi feito um teste preliminar no mês de junho de 2011, com 44 alunos dos grupos de teste que cursavam a disciplina Máquinas Elétricas em uma instituição federal de ensino, turno diurno, utilizando este experimento em sala de aula. Para o experimento foi utilizado um laptop com webcam e um datashow. Inicialmente os estudantes puderam observar o professor e posteriormente cada um pode manipular o Objeto de Aprendizagem.

\section{Como Funciona a Realidade Au- mentada}

A Realidade Aumentada, a partir de filmar uma cena em tempo real, e a partir de um marcador presente na cena, traz para a tela do computador uma cena em um mundo virtual misturado com um mundo real, que é o mundo em Realidade Aumentada. A formação do ambiente em Realidade Aumentada é exemplificada na figura 12.

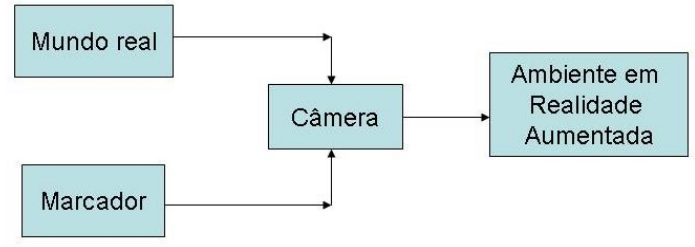

Figura 12 : Formação do mundo em Realidade Aumentada [21]

A Realidade Aumentada pode ser definida como "um sistema que suplementa o mundo real com objetos virtuais gerados por computador, com a impressão de coexistência no mesmo espaço" [22]. RA é a combinação de objetos reais e objetos virtuais. Objetos reais são dominantes e objetos virtuais são informação adicional [23].

Azuma [22] define RA como um sistema que tem as seguintes características:

1. Combina real e virtual;

2. É interativa com processamento em tempo real;

3. É concebida em três dimensões.

Para a criação do ambiente em Realidade Aumentada foi utilizado o ARToolKit (Augmented Reality Toolkit). De acordo com Coelho e Bähr [8], o ambiente em Realidade Aumentada é formado a partir da mistura da filmagem de um certo local com cenas de um mundo virtual.

A Realidade Aumentada funciona da seguinte maneira:

1-Coloca-se um marcador em um objeto onde se deseja que ocorra a interação;

2-Esse marcador será visualizado pela câmera do microcomputador;

3-Se o mesmo for reconhecido, levará a uma biblioteca pré-estabelecida;

4-Aparecerá, então, na tela do computador, o primeiro objeto onde estava o marcador, juntamente com o objeto que estará na biblioteca. 
5-Os dois objetos serão fundidos em um mundo misto que misturará o mundo real com o mundo virtual.

\section{Ferramenta utilizada para criação do ambiente em Realidade Aumen- tada}

O software ARToolKit (Augmented Reality Toolkit), que possui biblioteca, com código aberto e gratuita, é apropriado para desenvolver aplicações de Realidade Aumentada [24].

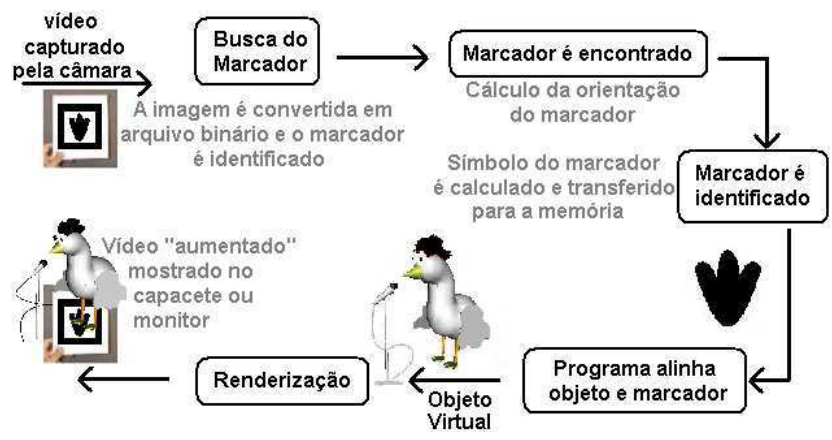

Figura 13 : Funcionamento básico do ARToolKit [23]

A figura 13 ilustra o funcionamento básico do ARToolKit.

O pacote disponibilizado contém bibliotecas para rastreamento e os códigos fonte destas bibliotecas para permitir que programadores adaptem para as suas necessidades. Disponível para fins não comerciais, o ARToolKit utiliza técnicas de visão computacional para calcular o ponto de vista da câmera em relação à marcadores quadrados que contém um padrão na parte interna, possibilitando o registro correto das imagens.

\section{Experimento utilizando RA}

O experimento com os alunos foi a demonstração do campo magnético girante de um motor. A RA foi aplicada ao estator de um motor elétrico, onde foram misturados o mundo real e o mundo virtual da seguinte maneira: a câmera filmou o motor e surgiu a imagem do mundo virtual, em que apareceram os campos magnéticos envolvidos, que no mundo real são invisíveis. O resultado disto foi uma imagem em RA: o motor e os campos magnéticos, neste caso, visíveis para o aluno, superando a grande dificuldade encontrada pelos estudantes, que é a visualização dos campos.

O motor elétrico utilizado neste experimento é o motor de corrente alternada assíncrono monofásico de fase dividida. Foi simulado o campo girante do estator.
Na figura 14 pode-se observar o estator utilizado no experimento.

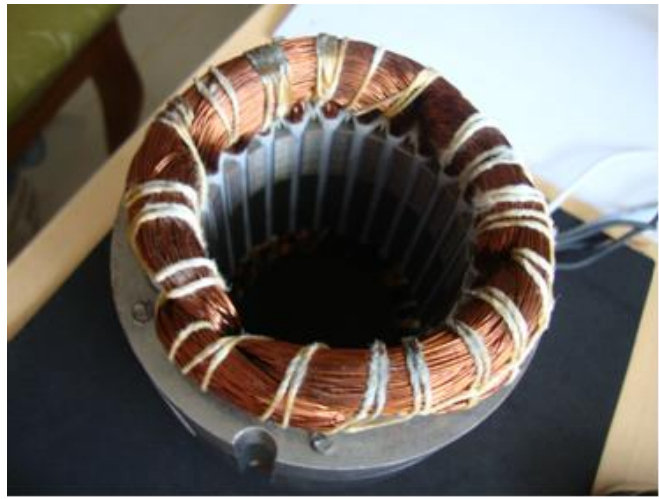

Figura 14 : Estator do motor em que foi realizado o experimento junto com o marcador

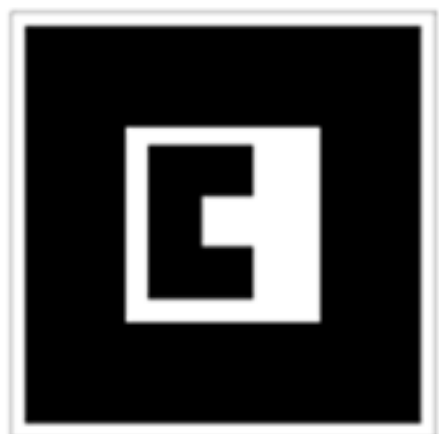

Figura 15 : Marcador utilizado no experimento

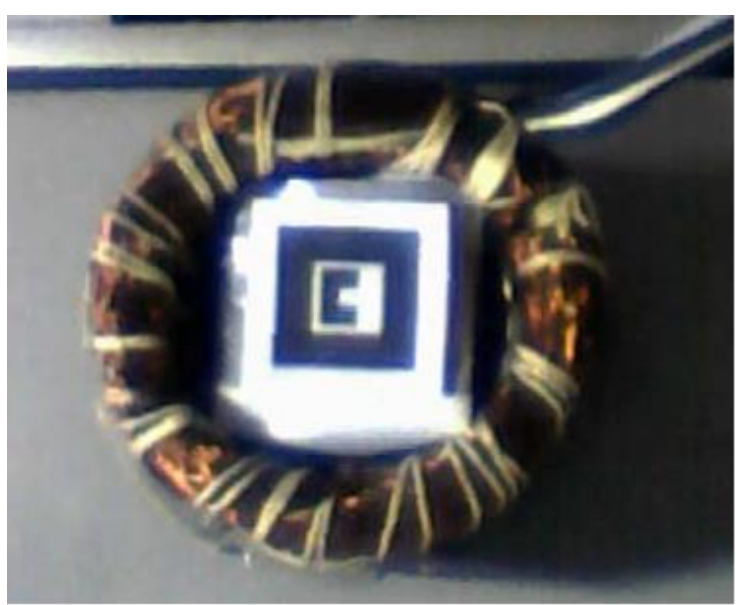

Figura 16 : Estator do motor em que foi realizado o experimento junto com o marcador

Este experimento foi desenvolvido com o software ARToolKit, pela facilidade de programação oferecida pelo mesmo. 
O marcador (figura 15) é colocado no centro do estator (mundo real), visto na figura 16, para que o seu campo magnético apareça (mundo virtual), formando o ambiente de RA.

Surge o campo girante do estator, que pode ser visto na figura 17. Na figura 18 pode-se ter uma idéia do campo girante, já que o mesmo está sendo mostrado em outra posição. As figuras 19 e 20 também mostram o campo girante em outras posições.

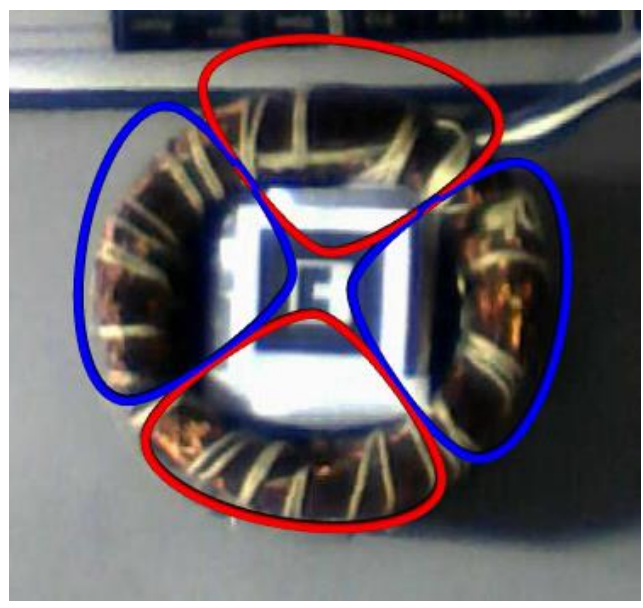

Figura 17 : Estator do motor junto com o campo girante - posição 1

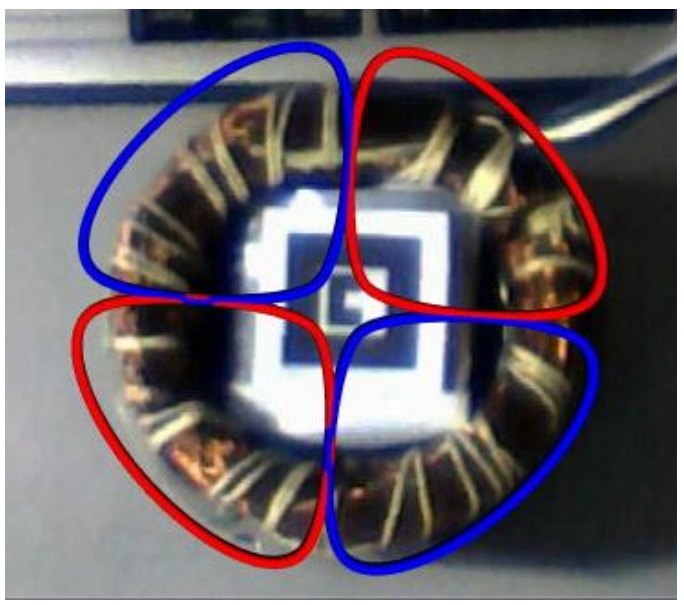

Figura 18 : Estator do motor junto com o campo girante - posição 2

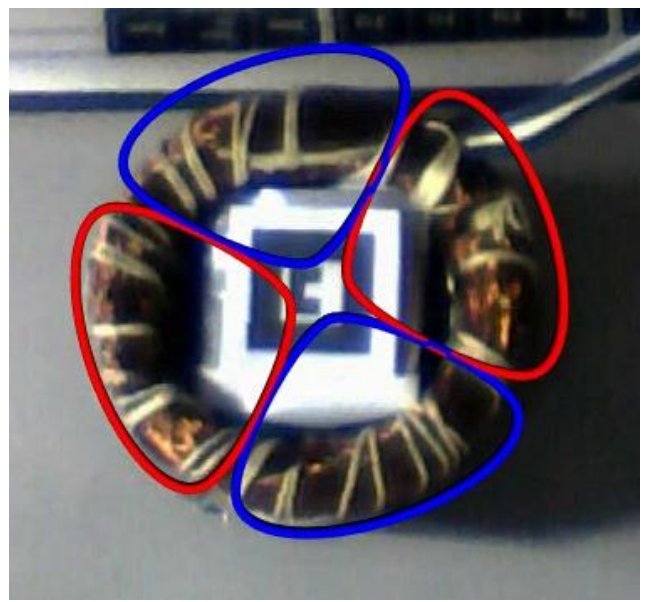

Figura 19 : Estator do motor junto com o campo girante - posição 3

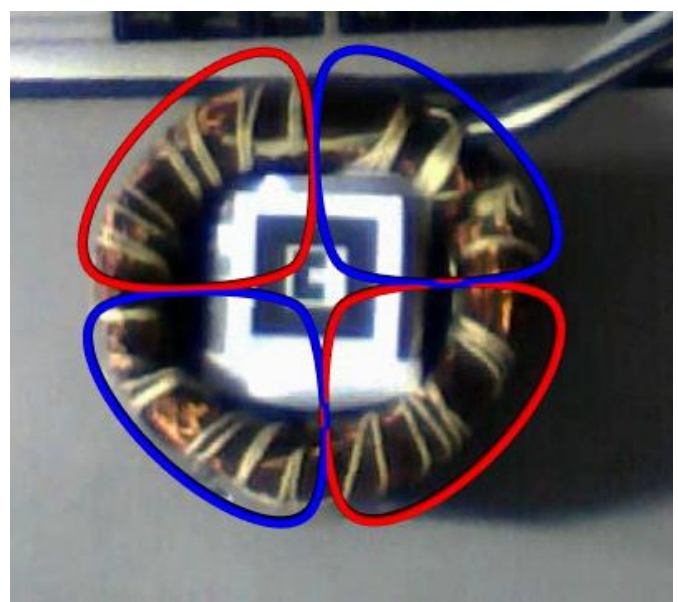

Figura 20 : Estator do motor junto com o campo girante - posição 4

Ainda neste mesmo experimento, o marcador agora fará outro link, com o objetivo de se visualizar o campo girante lateralmente (figura 21).

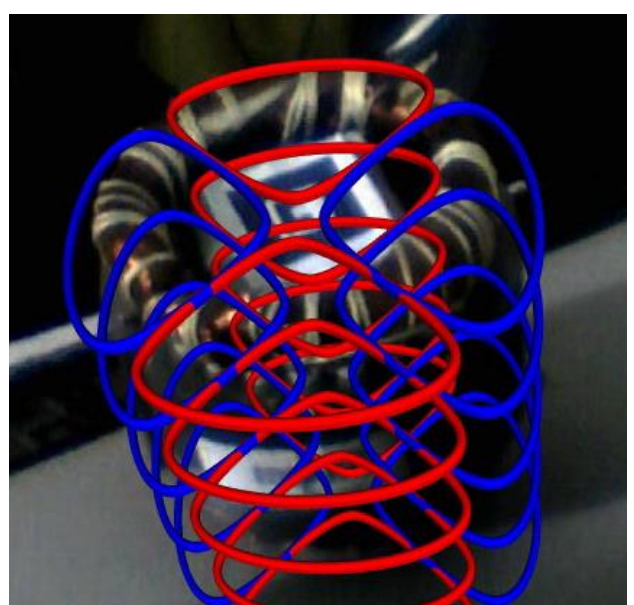

Figura 21 : Vista lateral do campo girante - posição 1 


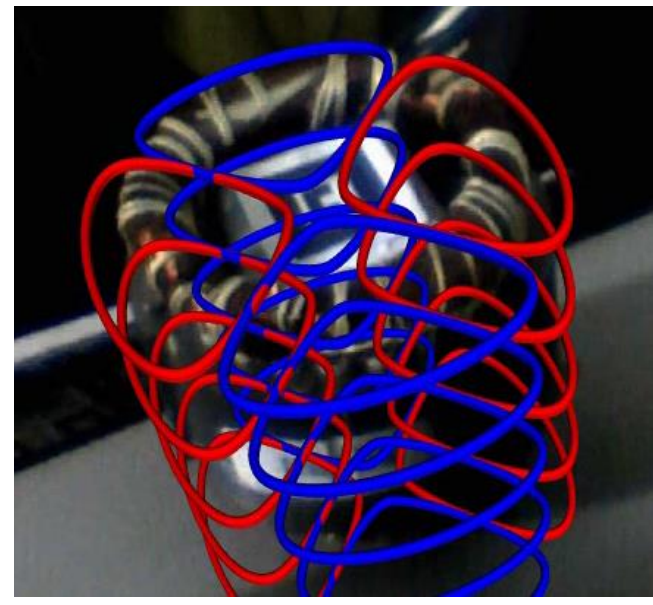

Figura 22 : Vista lateral do campo girante - posição 2

Surgirá, no mundo virtual, o campo girante visto da parte lateral do motor. Serão visualizados o motor e o seu campo girante em vista lateral em ambiente de RA. Nas figuras 22,23 e 24 pode-se observar o campo em outras posições.

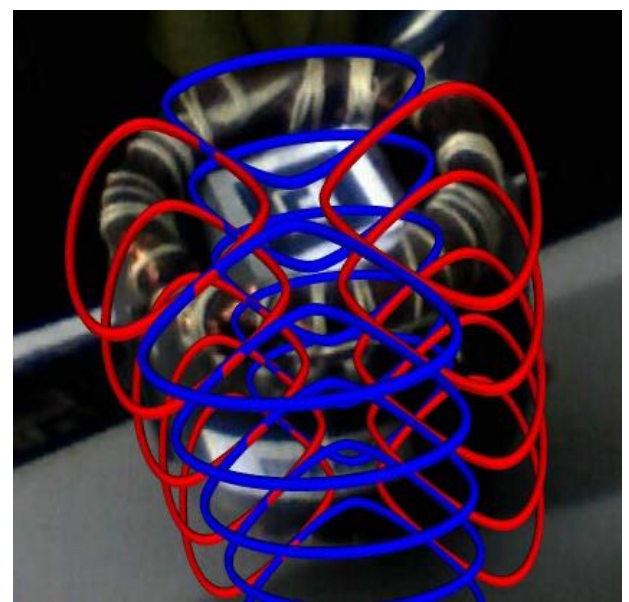

Figura 23 : Vista lateral do campo girante - posição 3

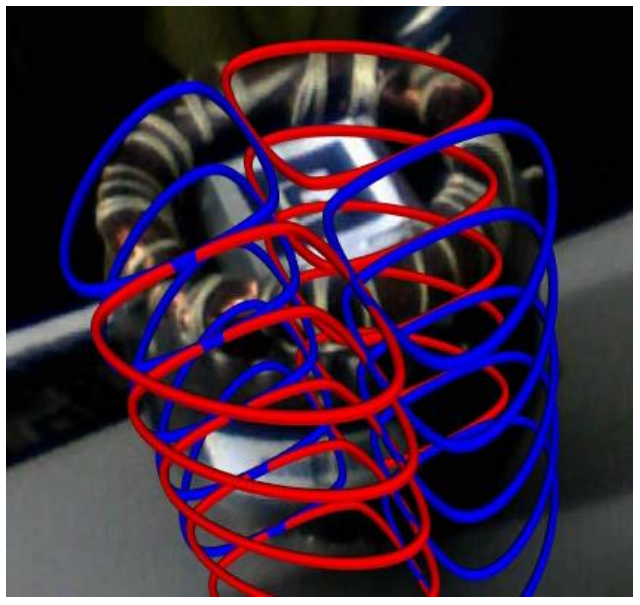

Figura 24 : Vista lateral do campo girante - posição 4

Ao final da aula responderam o Pós-teste para verificação da aprendizagem.

\section{Teste para verificação dos indícios da Aprendizagem Significativa}

De acordo com Moreira [19] uma maneira de se evitar a "simulação da Aprendizagem Significativa" é formular questões inéditas ao aprendiz. Moreira e Masini [18] propõem que para verificar os indícios da Aprendizagem Significativa seja colocada uma nova tarefa que dependa da precedente. A partir da aula sobre formação do campo girante foram realizadas as atividades do quadro 1 .

Foi realizado um pré-teste para verificação do estágio inicial dos estudantes em relação ao assunto a ser estudado. A partir da aula sobre formação do campo girante foram realizadas as atividades do quadro 1. Ao final, os estudantes responderam perguntas inéditas relacionadas ao novo conhecimento (Pós-teste). As perguntas do Pré-teste foram as mesmas perguntas do Pós-teste para posterior comparação. Foi feito um total de nove perguntas, sendo quatro sobre o conteúdo estudado e cinco sobre a percepção do aluno em relação ao estudo de Eletromagnetismo. São descritas aqui as três perguntas mais relevantes, as duas primeiras sobre a percepção do aluno e a terceira sobre o tema estudado. Os resultados são relativos ao Pré-teste e Pós-teste.

As atividades foram realizadas no curso de Eletrotécnica do Instituto Federal Fluminense em quatro turmas, três de teste, em que foi realizado o experimento com RA (turmas A, B e C), totalizando 44 alunos e um grupo de controle que não participou do experimento com RA (grupo D), com 17 alunos. 
Quadro 1 : Atividades realizadas

\begin{tabular}{|l|l|}
\hline \multicolumn{1}{|c|}{ Turmas de Teste } & Turmas de Controle \\
\hline $\begin{array}{l}\text { Pré-teste: Perguntas rela- } \\
\text { tivas ao assunto a ser } \\
\text { estudado e sobre a per- } \\
\text { cepção do aluno. }\end{array}$ & $\begin{array}{l}\text { Pré-teste: Perguntas } \\
\text { relativas ao assunto a } \\
\text { ser estudado e sobre a } \\
\text { percepção do aluno. }\end{array}$ \\
\hline $\begin{array}{l}\text { Explicação tradicional } \\
\text { do campo girante. }\end{array}$ & $\begin{array}{l}\text { Explicação tradicional } \\
\text { do campo girante. }\end{array}$ \\
\hline $\begin{array}{l}\text { Experimento utilizando } \\
\text { RA. }\end{array}$ & \multicolumn{1}{|c|}{ xxx } \\
\hline $\begin{array}{l}\text { Pós-Teste: Mesmas per- } \\
\text { guntas do Pré-teste. }\end{array}$ & $\begin{array}{l}\text { Pós-Teste: Mesmas } \\
\text { perguntas do Pré-teste. }\end{array}$ \\
\hline
\end{tabular}

\subsection{Teste: primeira pergunta}

A primeira pergunta foi: "Você achou fácil compreender os conceitos de eletromagnetismo?". As respostas dos alunos relativas ao Pré-teste e Pós-teste se encontram no gráfico 1 .

\subsubsection{Resultados: turmas de teste $(A+B+C)$ e turma de controle}

No pré-teste, $4,5 \%$ das turmas de teste afirmaram que acharam fácil compreender os conceitos de Eletromagnetismo, enquanto que no pós-teste, este número subiu para $52,3 \%$ (gráfico 1). Na turma de controle (D) nenhum aluno achou fácil compreender os conceitos de Eletromagnetismo no pré-teste e no pós-teste $14,3 \%$ dos alunos acharam fácil.

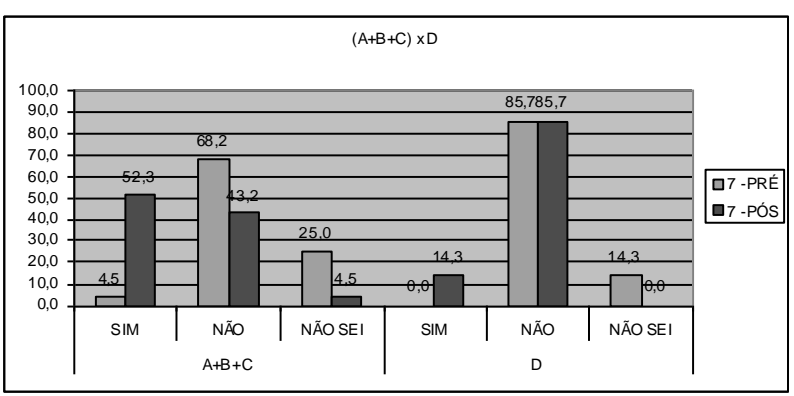

Gráfico 1 : Resposta dos alunos à pergunta $\mathrm{n}^{\mathrm{o}} 1$ Turmas de Teste $(\mathrm{A}+\mathrm{B}+\mathrm{C})$ e Turma de Controle (D) Porcentagem de alunos $\mathrm{X}$ sim / não / não sei

\subsubsection{Análise dos resultados: comparação entre as turmas de teste $(A+B+C)$ e a turma de controle (D)}

Comparando com o somatório das turmas de teste com a turma de controle, observa-se que os alunos das turmas de teste, que estudaram os conceitos de eletromagnetismo usando RA, tiveram uma percepção de facilidade na compreensão maior que os estudantes da turma de controle que não tiveram acesso a RA como ferramenta de ensino-aprendizagem, demonstrando a melhoria do processo ensino-aprendizagem quando se utilizou a RA.

\subsection{Teste: segunda pergunta}

A segunda pergunta foi: "Você achou fácil visualizar os campos magnéticos?". As respostas dos alunos se encontram no gráfico 2 .

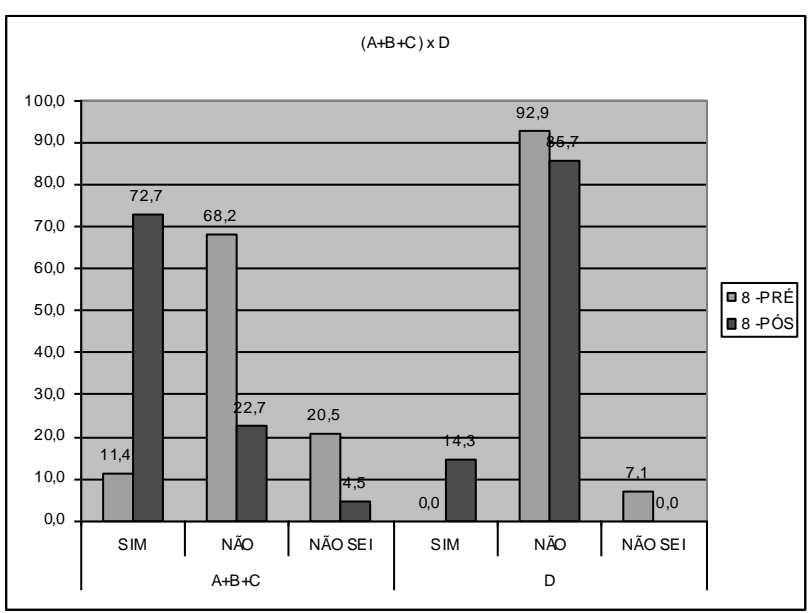

Gráfico 2 : Resposta dos alunos à pergunta $\mathrm{n}^{\circ} 2$ Turmas de Teste $(\mathrm{A}+\mathrm{B}+\mathrm{C})$ e Turma de Controle (D) Porcentagem de alunos X sim / não / não sei

\subsubsection{Resultados: turmas de teste $(A+B+C)$ e turma de controle}

Nas turmas de teste, no pré-teste, $11,4 \%$ dos alunos responderam que foi fácil visualizar os campos magnéticos, enquanto que no pós-teste, $72,7 \%$ dos alunos afirmaram que acharam fácil (gráfico 2). Já na turma de controle, nenhum aluno achou fácil no pré-teste, enquanto que $14,3 \%$ dos alunos acharam fácil.

\subsubsection{Análise dos resultados: comparação entre as turmas de teste $(A+B+C)$ e a turma de controle (D)}


As turmas de teste A, B e C, que utilizaram a RA no estudo de eletromagnetismo, tiveram percepção de facilidade de visualização dos campos magnéticos bem superior à turma de controle.

Considerando o nível de abstração envolvido na visualização dos campos, a RA foi uma importante ferramenta nesse estudo. Esta questão demonstra claramente a importância da RA neste estudo.

\subsection{Teste: terceira pergunta}

A terceira pergunta foi: "Explique o princípio de funcionamento do gerador elementar". As respostas dos alunos se encontram no gráfico 3 para as turmas de teste comparadas com a turma de controle.

\subsubsection{Resultados: turmas de teste $(A+B+C)$ e turma de controle}

No pré-teste, nas turmas de teste, $2,3 \%$ dos alunos sabiam o princípio do funcionamento do gerador elementar (gráfico 3). No pós-teste, 54,5\% sabiam. Na turma de controle (D), no pré-teste nenhum aluno sabia, enquanto que no pós-teste $14,3 \%$ dos alunos sabiam.

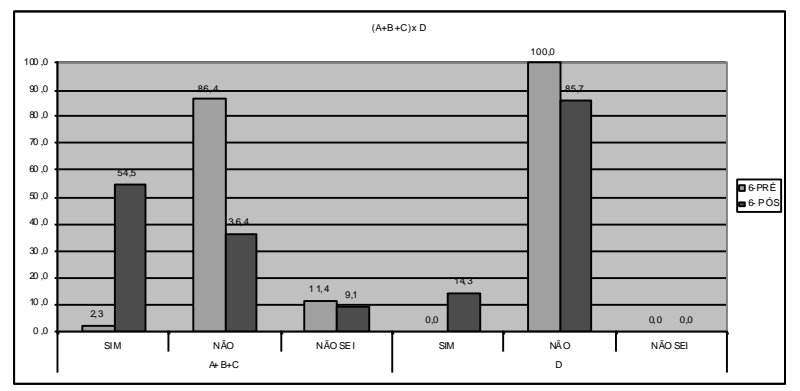

Gráfico 3 : Resposta dos alunos à pergunta $n^{0} 3$

Turmas de Teste $(\mathrm{A}+\mathrm{B}+\mathrm{C})$ e Turma de Controle (D)

Porcentagem de alunos X sim / não / não sei

\subsubsection{Análise dos resultados: comparação entre as turmas de teste $(A+B+C)$ e a turma de controle (D)}

Confrontando os resultados do somatório das turmas teste com a de controle, observa-se que $54 \%$ dos estudantes das turmas de teste aprenderam o princípio de funcionamento do gerador, enquanto que na turma de controle o êxito foi de apenas $14 \%$ evidenciando a facilitação do aprendizado proposta pelo recurso da RA neste estudo.

\section{Conclusões}

Tendo em vista que um dos grandes problemas apontados pelos professores no processo ensino-aprendizagem de Eletromagnetismo é a dificuldade por parte do aluno na visualização dos campos magnéticos, esta pesquisa investigou se, utilizando a RA como ferramenta no processo ensino-aprendizagem de Eletromagnetismo houve melhoria no entendimento desse assunto por parte do aluno.

Este trabalho visou investigar se a visualização e a interação proporcionadas pela Realidade Aumentada, utilizada como ferramenta no processo ensino-aprendizagem, contribuiu para o processo ensino-aprendizagem no ensino de campos magnéticos girantes.

Foi estruturada uma atividade pedagógica utilizando RA como ferramenta no processo de ensinoaprendizagem. Foram testados os resultados da utilização desta ferramenta como apoio neste processo. Também foram analisados os resultados que apontaram as evidências da aprendizagem utilizando-se o OA construído em ambiente de RA a partir da Teoria da Aprendizagem Significativa.

A Realidade Aumentada apresentou as seguintes vantagens: permitiu aos alunos a visualização do campo magnético girante; permitiu a interação do aluno com estes campos magnéticos; a partir do momento em que o Objeto de Aprendizagem em Realidade Aumentada foi elaborado, sua utilização é simples e prática; simplicidade e economia do equipamento utilizado (os experimentos foram realizados com apenas uma webcam e um laptop); foi utilizado também um data show com telão, sendo que o data show não é necessário, servindo apenas para enriquecer o experimento; o ambiente em Realidade Aumentada é excelente para visualização dos campos magnéticos.

Os resultados mostram que houve melhorias estatisticamente significativas no desempenho dos aprendizes do grupo experimental, quando comparados aos aprendizes do grupo de controle. Neste trabalho foi proposta uma nova forma de ensinar o campo magnético girante. Com este experimento, e de acordo com o teste respondido pelos alunos, pode-se verificar que a Realidade Aumentada pode contribuir como ferramenta no processo de ensino-aprendizagem do campo girante a partir da Teoria da Aprendizagem Significativa.

\section{Referências}

A. M. Paz, Atividades Experimentais e Informatizadas: Contribuições para o Ensino de Eletromagnetismo. Tese de Doutorado (Programa de Pós-Graduação em Educação Científica e Tecnológica), Universidade Federal de Santa 
Catarina, 2007.

[2] S. H. Macedo, F. A. Fernandes, J. V. Lima, M. C. V. Biazus. Ensino e Aprendizagem do Campo Magnético de um Imã Usando Realidade $\mathrm{Au}-$ mentada. In anais do XXXIX COBENGE - Congresso Brasileiro de Educação em Engenharia, Blumenau, 2011.

[3] A. Buchau, W. M. Rucker, U. Wössner, M. Becker. Augmented Reality in Teaching Eletrodynamics. In The International Journal for Computation and Mathematics in Electrical and Electronic Engineering. V. 28, n. 4, páginas 948963, 2009.

[4] W. A. Silva, M. W. S Ribeiro, E. Lamounier Jr., A. Cardoso. Uma Arquitetura para Distribuição de Ambientes Virtuais de Realidade Aumentada aplicada à Educação. RBIE. Revista Brasileira de Infomática na Educação. V. 16. n. 3. Set-dez 2008.

[5] A. D. Wiley. Connecting learning objects to instructional design theory: A definition, a metaphor, and a taxonomy. In D. A. Wiley (Ed.), The Instructional Use of Learning Objects: Online Version. 2000. Disponível em: http://reusability.org/read/chapters/wiley.doc. Acesso em 15/02/2012.

[6] N. M. Barros. Aprendizagem a Distância. Do Rádio Ilustrado à Realidade Virtual Aumentada. Florianópolis, SC, Insular. 2007.

[7] S. H. Macedo, E. S. Leite. "Estudo Sobre o Software Electronics Workbench como Ferramenta de Ensino-aprendizagem de Eletrônica Digital". In $21^{\circ}$ Simpósio Brasileiro de Informática na Educação. SBIE, 2010.

[8] A. H. Coelho, H. P. Bähr. Visualização de dados de CAD e LIDAR por meio de Realidade Aumentada. In: XII Simpósio de Sensoriamento Remoto, INPE, pp. 2925-2932. Abril 2005. Disponível em:

$<$ http://marte.dpi.inpe.br/col/ltid.inpe.br/sbsr/200 4/11.11.08.15/doc/2925.pdf $>\quad$ Acesso em 22/05/2010.

[9] H. Kaufmann, K. Steinbüegl, A. Dünser, J. Glück,. General Training of Spatial Abilities by Geometry Education in Augmented Reality. In: Annual Review of CyberTherapy and Telemedi- cine: A Decade of $V R$, vol. 3, páginas 65-76, 2005. Disponível em:

<http://www.ims.tuwien.ac.at/media/documents/ publicatins/CT05_GeomEdu_SpatialAbilities. pdf> Acesso em 27/07/2010.

[10] E. Fernandes. $5^{a}$ Aula de Máquinas Elétricas CA. 2010. Disponível em:

http://emannuelfernandes.blogspot.com/2010_08 _01_archive.html

[11] J. A. Valente. Diferentes Usos do Computador na Educação. Em Aberto, n. 57. Jan-mar 1993.

[12] M. A. Moreira, E. F. S. Masini. Aprendizagem Significativa - A Teoria de David Ausubel. Centauro, São Paulo, 2001.

[13] C. C. Guimarães. "Experimentação no ensino de Química: Caminhos e Descaminhos rumo à Aprendizagem Significativa", Química Nova na Escola. Vol. 31, agosto, 2009.

[14] S. H. Macedo. Estudo de Sólidos Utilizando Recursos de Realidade Aumentada: uma Experiência em Sala de Aula. XVI ENDIPE - Encontro Nacional de Didática e Práticas de Ensino. Campinas. UNICAMP, 2012. v.1. p. 1-10. 2012.

[15] R. AZUMA. A Survey of Augmented Reality. 1995. Disponível in:

http://www.cs.unc.edu/ azuma/ARpresence.pdf

[16] P. Milgram; F. Kishino. A Taxonomy of Mixed Reality Visual Displays. 1994. IEICE Transactions on Information and Systems. Disponível em:

$<$ http://etclab.mie.utoronto.ca/people/paul_dir/IE ICE94/ieice.html>. Acesso em: 24/jul/2010.

[17] Ezequiel Roberto Zorzal. Estratégia para o Desenvolvimento de Aplicações Adaptativas de Visualização de Informações com Realidade Aumentada. 2009. 174 p. Tese de Doutorado (Programa de Pós-graduação em Engenharia Elétrica). Universidade Federal de Uberlândia.

[18] M. A. Moreira. A teoria da Aprendizagem Significativa e sua implementação em sala de aula. Editora Universidade de Brasília, Brasília, 2006.

[19] M. A. Moreira, E. F. S. Masini. Aprendizagem Significativa - A Teoria de David Ausubel. Centauro, São Paulo, 2001. 
